\title{
Measurement and Adaptive Identification of Nonstationary Acoustic Impulse Responses
}

\author{
M. Mekarzia (iD \\ Department of Aeronautics, University of Blida, Algeria \\ Correspondence should be addressed to M. Mekarzia; m_mekarzia@yahoo.fr
}

Received 7 July 2018; Accepted 19 June 2019; Published 16 July 2019

Academic Editor: Kim M. Liew

Copyright (c) 2019 M. Mekarzia. This is an open access article distributed under the Creative Commons Attribution License, which permits unrestricted use, distribution, and reproduction in any medium, provided the original work is properly cited.

In this work, we present a method of measurement of nonstationary acoustic impulse responses identified by the fast version of the Recursive Least Squares algorithm (FRLS), using professional acoustic equipment. This measurement bench realized in a deaf room presents several tests of capability of adaptive algorithm to tracking the nonstationarities of true system to be identified. The tests of tracking capability obtained are stronger compared to what is encountered in real life and can be used in several applications.

\section{Introduction}

Acoustic phenomena in an acoustic medium are measured from the notion of the acoustic channel (Figure 1), which depends on three main elements:

(i) The shape and acoustic properties of the walls and objects in the room

(ii) The source with its spectral emission diagram and disposition (LS: loudspeaker)

(iii) The receiver with its directivity diagram and its position (MIC: microphone)

In the approximation of linear acoustics, this channel is a linear filter whose input is the signal $\mathrm{x}(\mathrm{t})$ and whose output is $y(t)$ :

$$
\mathrm{y}(\mathrm{t})=\mathrm{h}(\mathrm{t}) * \mathrm{x}(\mathrm{t})
$$

where $h(t)$ is the impulse response (IR) of the acoustic channels $[1,2]$.

The purpose of the measurement is to find the (IR) $\mathrm{h}(\mathrm{t})$ which completely characterizes the acoustic channel. In acoustic rooms, these IR have a duration of the order of one second, and the desired dynamics is of the order of $60 \mathrm{~dB}$. The conventional measurement technique that generates an impulse and records the RI does not allow to simultaneously meet all these requirements $[1,2]$.
In addition, the risk increases considerably when the transducers (LS and MIC) are pulsed. We describe the measurement method that is better adapted to the requirements of IR measurement. We can apply this method to the impulse response measurement of the individual head [1].

\section{Impulse Response of the Individual Head}

To measure individual impulse responses of the head (Figure 2) which allow to obtain a better sound spatial restitution for the noninvasive sensory substitution aid for blind people by using the auditory pathway for the representation of the frontal visual stage real time, we must take into account the following:

(i) Synthesis of echoes to restore the distance

(ii) Minimal time analysis between two sounds

$S$ : it is the source in terms of signal $x(t)$.

Ear: it is the receiver in terms of signal $y(t)$ [3].

\section{Utilized Equipment}

The equipment used in the test bench is described as follows:

(i) Two-channel real-time frequency analyzer of type 2133B \& K (B \& $K=$ Bruel \& Kjaer). We used the latter to, on the one hand, generate the excitation 


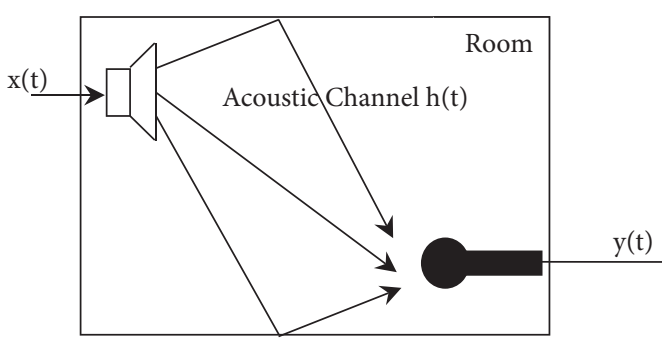

FIgURE 1: Acoustic channel [1].

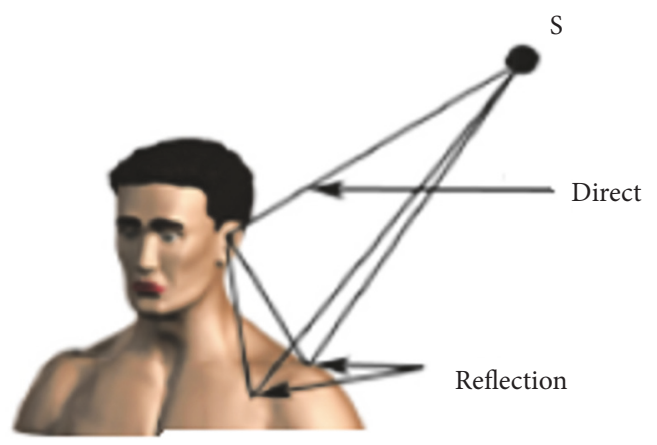

FIGURE 2: Example of application [3].

signal which is a pseudorandom white noise $\mathrm{x}$ (n) of frequency band equal to $5 \mathrm{KHz}$ and amplitude 2 volts effective. On the other hand, this analyzer allows the acquisition in real time and in synchronism with the excitation $\mathrm{x}(\mathrm{n})$ of the response signal of the room $\mathrm{y}(\mathrm{n})$ $[1,4]$.

(ii) A loudspeaker of type 4224B \& $\mathrm{K}$ that transforms the excitation signal $\mathrm{x}(\mathrm{n})$ into a sound pressure that will be broadcast in the room $[1,5]$.

(iii) A rotating arm microphone of type 3923B \& K that converts acoustic pressures into electrical signals usable on the spectrum analyzer.

The microphone-speaker pair defines the acoustic channel of the room $[1,6]$.

(iv) A precision integrating modular sound meter of type 2131B \& $\mathrm{K}$ that measures the level of ambient noise in the room during measurement $[1,7]$.

The measurement of the impulse response of the acoustic channel is carried out in two steps:

(i) Synchronous acquisition of the signals $x(n)$ and $y(n)$ with the equipment described above.

(ii) Identification in deferred time of the impulse response.

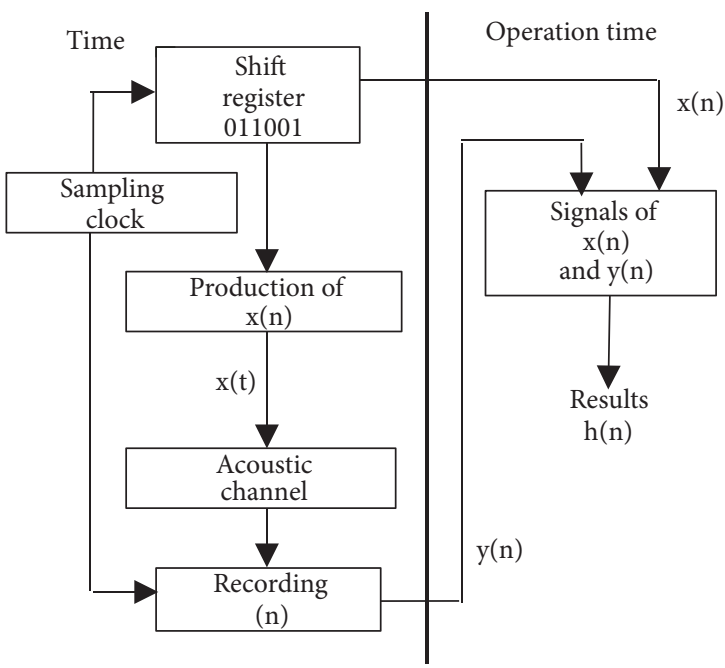

FIgURE 3: Flowchart of the measurement method [1].

\section{Principle of Measurement of Impulse Responses}

The general flowchart of the measurement method is given in Figure 3. The main element of this method is the identification of the impulse response $h(t)$ from the two signals $x(t)$ and $y(t)[1,8]$.

\section{Table of Fast Recursive Leas Squares Algorithm (FRLS) [9]}

(i) Proper choice of $\mu^{\gamma} \cdot \mu^{\beta} \cdot \mu^{\alpha}$ and $\lambda$

(ii) Variables available at the moment t:

$$
a_{N, t-1}, b_{N, t-1}, \bar{\complement}_{N, t-1}, \gamma_{N, t-1}, \alpha_{N, t-1}, \beta_{N, t-1}, H_{N, t-1}
$$

(iii) New information: $x_{t}$ and $y_{t}$

(iv) Modeling of $x_{t}$ and $x_{t-N}$ :

$$
\begin{aligned}
\bar{e}_{N, t} & =x_{t}-a_{N, t-1}^{T} X_{N, t-1} \\
\alpha_{\mathrm{N}, \mathrm{t}} & =\lambda \alpha_{\mathrm{N}, \mathrm{t}-1}+\gamma_{\mathrm{N}, \mathrm{t}-1} \overline{\mathrm{e}}_{\mathrm{N}, \mathrm{t}}^{2} \\
\gamma_{N+1, t} & =\frac{\lambda \alpha_{N, t-1}}{\alpha_{N, t}} \gamma_{N, t-1} \\
\bar{c}_{N+1, t} & =\left[\begin{array}{c}
0 \\
\bar{c}_{N, t-1}
\end{array}\right]-\frac{\bar{e}_{N, t}}{\lambda \alpha_{N, t-1}}\left[\begin{array}{c}
1 \\
-\alpha_{N, t-1}
\end{array}\right] \\
a_{N, t} & =a_{N, t-1}-\bar{e}_{N, t} \gamma_{N, t-1} \bar{c}_{N, t-1} \\
\bar{r}_{N, t} & =x_{t-N}-b_{N, t-1}^{T} X_{N, t}
\end{aligned}
$$




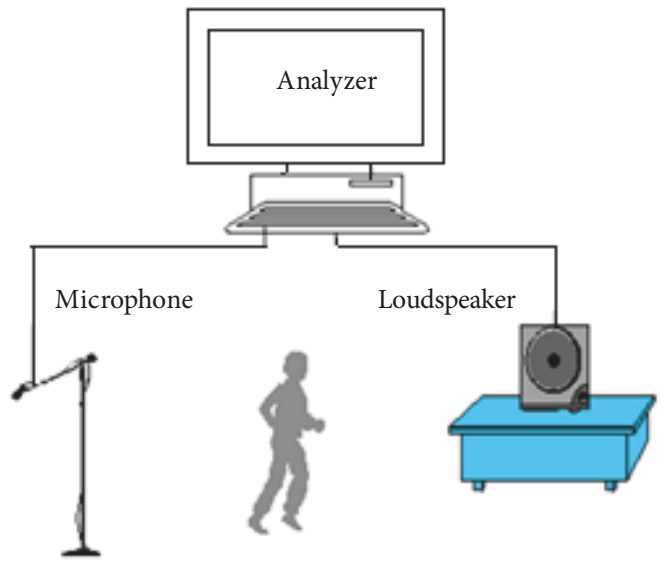

FIGURE 4: Room configuration during measure experiments [3].

$$
\begin{aligned}
& \xi_{N, t}=\bar{r}_{N, t}+\lambda \beta_{N, t-1} \bar{c}_{N+1, t}^{N+1} \\
& \bar{r}_{N, t}^{\gamma}=\bar{r}_{N, t}+\mu^{\gamma} \xi_{N, t} \\
& \bar{r}_{N, t}^{\beta}=\bar{r}_{N, t}+\mu^{\beta} \xi_{N, t} \\
& \bar{r}_{N, t}^{b}=\bar{r}_{N, t}+\mu^{b} \xi_{N, t} \\
& {\left[\begin{array}{c}
\bar{c}_{N, t-1} \\
0
\end{array}\right]=\bar{c}_{N+1, t}-\bar{c}_{N+1, t}^{N+1}\left[\begin{array}{c}
-b_{N, t-1} \\
1
\end{array}\right]} \\
& b_{N, t}=b_{N, t-1}-\bar{r}_{N, t}^{b} \gamma_{N, t} \bar{c}_{N, t} \\
& \beta_{N, t}=\lambda \beta_{N, t-1}+\gamma_{N, t}\left(\begin{array}{c}
-\beta \\
r_{N, t}
\end{array}\right)^{2}
\end{aligned}
$$

(v) Filtering of $y_{t}$ :

$$
\begin{aligned}
\varepsilon_{N, t} & =y_{t}-H_{N, t-1}^{T} X_{N, t} \\
H_{N, t} & =H_{N, t-1}-\bar{\varepsilon}_{N, t} \gamma_{N, t} \bar{c}_{N, t}
\end{aligned}
$$

\section{Description of the Different Measurements Taken}

Our measurements were taken in a room 20 meters long, 8 meters wide, and 3.5 meters high. The sound system during the measurements consists of a rotating arm on which are mounted the microphone and a speaker that radiates in the direction of the microphone (Figure 4).

The acoustic changes in the room or nonstationary acoustic channel coupling are caused randomly by the movement of a person between the microphone and the speaker. Depending on the speed of motion of the person, the acoustic changes are considered over time as follows: Slow, Medium, and Fast. The movements of the person between the microphone and the speaker introduce nonstationarities of acoustic channel that can be considered strong compared to those encountered in real-life situations.
The excitation signal $\mathrm{x}(\mathrm{n})$ of the loudspeaker (of type 4224) is a stationary pink noise generated by the spectrum analyzer (of type 2133) whose sampling frequency is $\mathrm{fs}=16$ $\mathrm{kHz}$.

The two signals $\mathrm{x}(\mathrm{n})$ and $\mathrm{y}(\mathrm{n})$ are separated and stored on disk in separate files for further processing. The nonstationarity of the acoustic channel results in nonstationarity in the signal y (n) picked up by the microphone. To check for nonstationarities caused by moving a person into the room, we have identified the signal $y(n)$ from the signal $x(n)$ using the fast recursive least squares algorithm (RLS) with exponential oblivion factor $\lambda$. An oblivion factor other than 1 allows the algorithm to continue tracking the nonstationarities of the acoustic channel. The identification error is given by

$$
\bar{\varepsilon}(n)=y(n)-H_{N}^{T} x(n)
$$

where the adaptation of $\mathrm{H}$ is carried out by RLS algorithm with an exponential oblivion factor $\lambda=0.9998$ and a size of the impulse response equal to 2500 points allows the algorithm to track the nonstationarities of the acoustic channel.

The performance criterion used is the time evolution energy of the normalized error $(y(n)-\widehat{y}(n))$ by the energy of the signal.

This criterion is given by

$$
J(n)=10 \cdot \log _{10}\left\langle\left\langle\bar{\varepsilon}_{N}^{2}\right\rangle /\left\langle y^{2}(n)\right\rangle\right\rangle
$$

where $<>$ is a short-term time average over a number of consecutive samples $(128,256 \ldots)$ [3].

This performance index is widely used in adaptive cancellation of acoustic echo [1].

\section{Simulation Results and Comments}

The results obtained in the case of a nonstationary acoustic channel are shown in Figures 5, 6, and 8. The size of the impulse response of the acoustic channel is $\mathrm{N}=2500$. The distance " $\mathrm{d}$ " between the microphone and the loudspeaker, the nature of the excitation signal, and the type of nonstationarity are also indicated.

In these figures, the nonstationarities of the acoustic channel appear as an increase in the value of the criterion $J(n)$.

In the stationary case, the $J(n)$ criterion regularly decreases towards a minimum value. Here the signal $x(n)$ is stationary; therefore the increase of the criterion $J(n)$ is due to an increase of the energy of the error $\epsilon_{N}(n)$. This increase in error is due to the inability of the adaptive algorithm to perfectly track nonstationarities in the acoustic channel. Therefore, the visible lobes on the temporal evolutions of $J(n)$ are the nonstationarities caused by the moving person in the room. The analysis of these results shows that the notion of the temporal rapidity or temporal slowness of the nonstationarity of the experimenter is not the same at the scale of the adaptive identification algorithm. This rapid or slow mobility of the experimenter results in an increase or decrease in the number of energy lobes in the evolution of the criterion $J(n)$. For the adaptive algorithm, all these results 


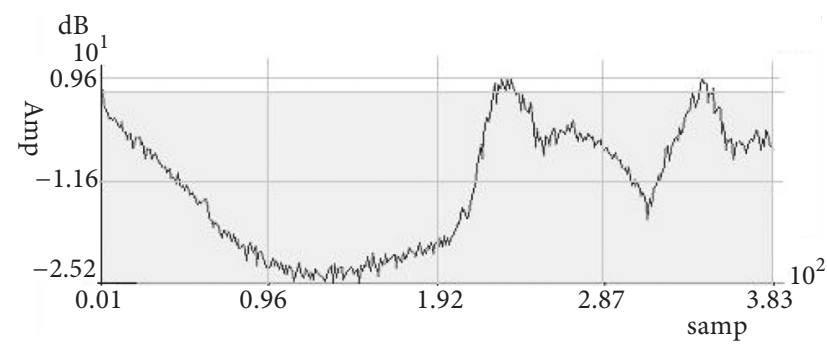

(a)

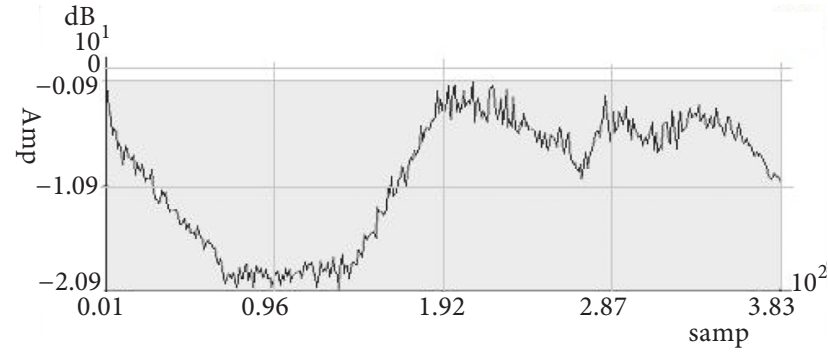

(b)

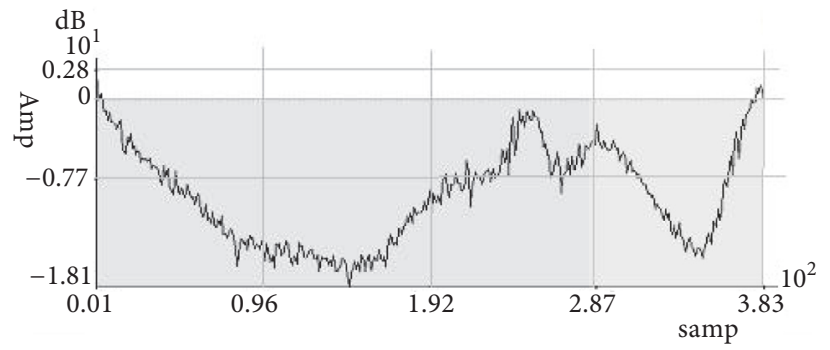

(c)

FIGURE 5: (a) Test of the tracking capacity algorithm with slow variations. (b) Test of the tracking capacity algorithm with medium variations. (c) Test of the tracking capacity algorithm with rapid variations [10].

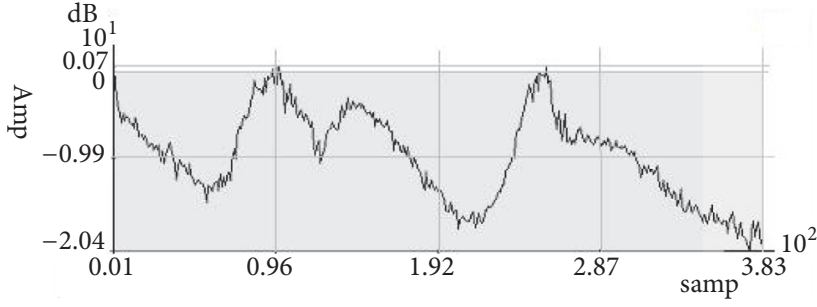

(a)

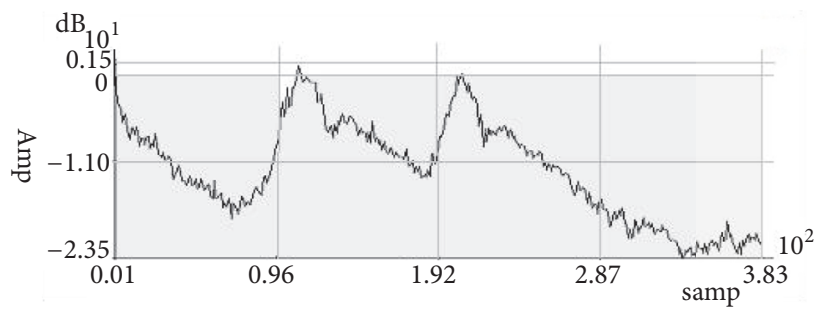

(b)

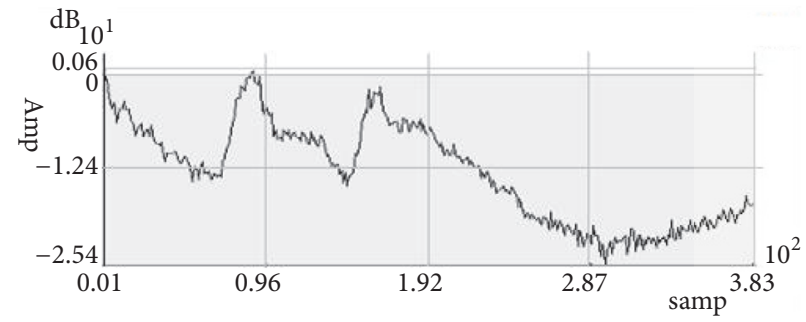

(c)

FIGURE 6: Nonstationary tests. (a) Near in time with slow variations. (b) With stationary final state with medium variations. (c) Near to an initial state with rapid variations [10].

can be considered as fast as the algorithm cannot perfectly follow the nonstationarities in the acoustic channel slowly or rapidly variable at the scale of the experimenter. Therefore, one can say that an adaptive algorithm is more successful in nonstationary situations when it gives the lowest maxima possible in $J(n)$ (lobe maxima).

For the use of the measurements carried out in the tests of the tracking capacity of adaptive algorithms we propose the following signals according to the type of the desired tests: (i) The tracking capacity close to an initial state: the signals do not leave enough time for the algorithm to converge from an initial state.

(ii) The ability of tracking from a permanent state: the signals do not leave enough time for the algorithm to reach an acceptable steady state.

(iii) Nonstationarities close in time: the signals explain that the lobes of nonstationarities caused by the person are close to each other. 


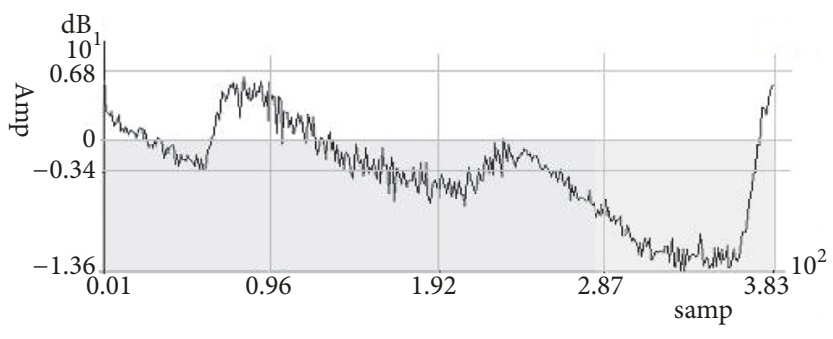

(a)

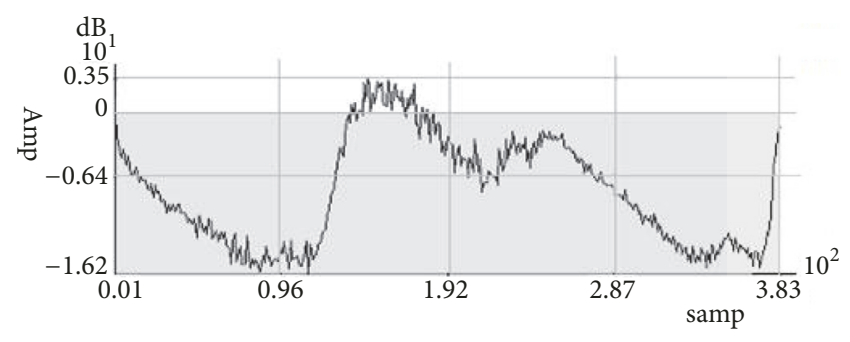

(b)

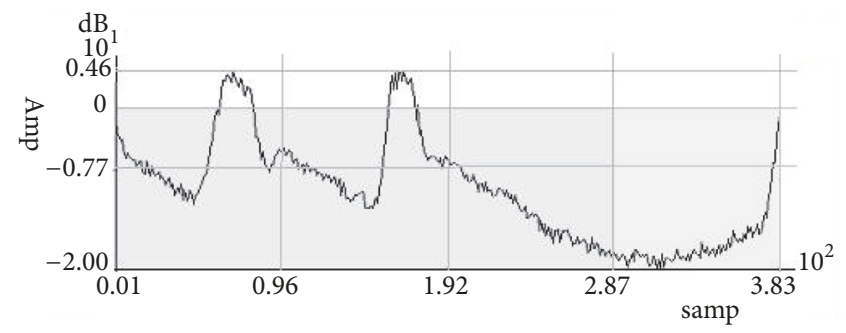

(c)

FIgURE 7: Nonstationary tests. (a) Near to an initial state with slow variations. (b) With stationary permanent state with medium variations. (c) Near in time with rapid variations [10].

(iv) Nonstationarities with a stationary final state: this type of signals makes it possible to give an estimate of the dynamics of the nonstationarity with respect to the stationary state.

In all the manipulations done in the laboratory, we used the same two-channel frequency analyzer (of type $2133 \mathrm{~B} \& \mathrm{~K}$ ), as well as the same acoustic medium, but we tested several pairs of microphones and loudspeakers. We realized a bench of measurement of nonstationary acoustic impulse responses. We quote some practical impulse responses obtained with their own parameters:

(i) The first experiment with the following measurement parameters:
(a) A loudspeaker of type 4224 B \& K
(b) A distance of $1.5 \mathrm{~m}$
(c) $x(n)$ that is a pink noise sequence

Convergence curves in this realistic context clearly show that we have given the algorithm time to converge and we have caused nonstationarity during the asymptotic phase. For this case we have good convergence and better tracking (Figure 5).

(ii) The second experiment with the following measurement parameters:
(a) A loudspeaker of type 4224 B \& K
(b) A distance of $2 \mathrm{~m}$
(c) $x(n)$ that is a pink noise sequence

In this experiment, we have two nonstationary lobes which aim to test the efficiency and robustness of the identification algorithms to track nonstationary systems close in time. We have obtained in this case a better tracking (Figure 6).

(iii) The third experiment with the following measurement parameters:
(a) A loudspeaker of type MICDIS
(b) A distance of $1 \mathrm{~m}$
(b) $x(n)$ that is a pink noise sequence

In this nonstationary acoustic impulse response measurement test, we have not given the algorithm enough time to converge. We have created two nonstationary lobes (tests (a) and (c)). The signal of test (b) gives the algorithm sufficient time to reach an acceptable steady state (Figure 7).

(iv) The fourth experiment with the following measurement parameters:
(a) A loudspeaker of type MICDIS
(b) A distance of $1.5 \mathrm{~m}$
(c) $x(n)$ that is a pink noise sequence

In this measurement test we have several lobes of system nonstationarities, which give the researcher the choice of nonstationarities to test their identification algorithms. On the other hand, these system nonstationarities may allow him to see the utility of a professional speaker and consumer speakers that poorly represent the low frequencies (Figure 8).

\section{Conclusion}

The analysis of these results shows that the notion of time speed or time slowness of the experimenter nonstationarity is not the same at the scale of the adaptive identification 


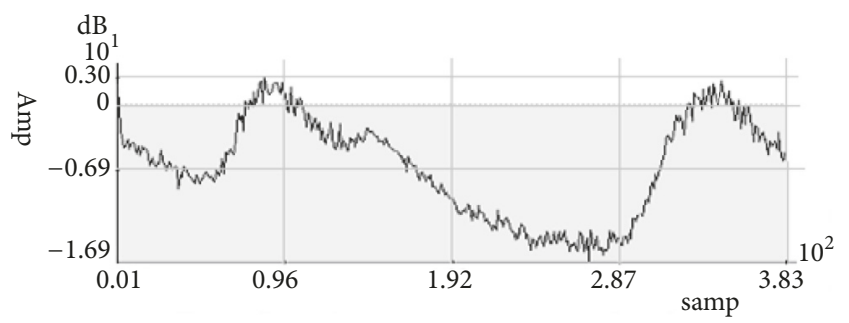

(a)

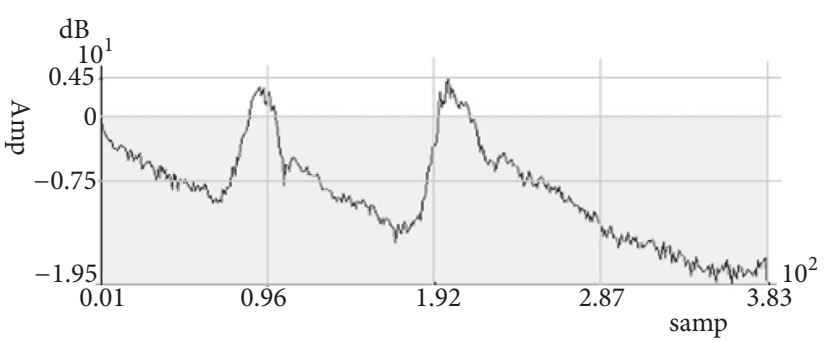

(b)

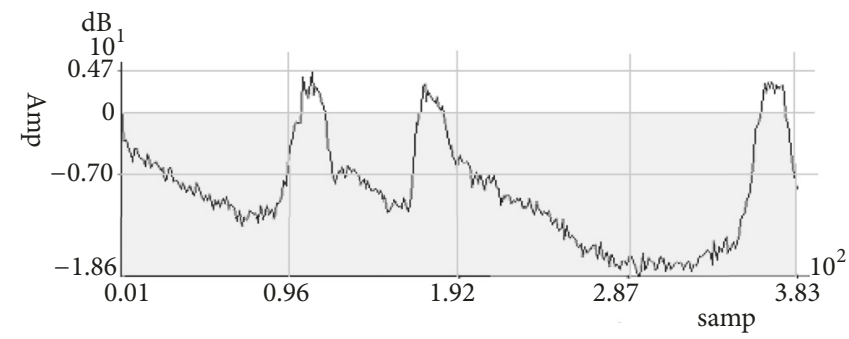

(c)

Figure 8: Nonstationary tests. (a) Far in time with slow variations. (b) Near to an initial state with medium variations. (c) Near in time with rapid variations $[10]$.

algorithm. This rapid or slow mobility of the experimenter leads to an increase or a decrease in the number of energy lobes in the evolution of the $J(n)$ criterion. As far as the adaptive algorithm is concerned, all these results can be considered as rapid since the algorithm cannot perfectly follow the nonstationarity in the acoustic channel slowly or rapidly variable at the experimenter scale.

Therefore, one can say that an adaptive algorithm tracks better nonstationarities when it gives the lowest maximums possible in $J(n)$ (lobe maxima).

\section{Data Availability}

The data used to support the findings of this study are available from the corresponding author upon request.

\section{Conflicts of Interest}

The author declares that he has no conflicts of interest.

\section{References}

[1] M. Mekarzia, "Measure and identification of acoustic impulse responses by NLMS-DC," 2007.

[2] M. Mekarzia, Measure And Identification of the Acoustic Impulse Responses, Institute of Aeronautics and Space Studies University of Blida, 2000.

[3] M. Mekarzia, Measure and Identification of the acoustic Impulse responses, Thesis of doctorate February, polythechnic school El Harrach Algerie, 2010.

[4] Analyzer of the frequencies real time 2123/2133 instruction, Analyzer of the frequencies real time 2123/2133 instruction, vol. 2, Brüel \& Kajaer, 1991.

[5] Loudspeaker 4224 Data, Brüel \& Kajaer, 1991.
[6] "Condenser microphone and microphone preamplifier for acoustic measurement," in Data Handbook, Brüel \& Kajaer, 1992.

[7] Sound Level Meter Type 2131 for Acoustic Measurement, Brüel \& Kajaer, 1992.

[8] M. Mekarzia and M. Guerti, "Measurement and identification of an acoustic impulse responses," Journal Building Acoustics, vol. 15, no. 1, pp. 73-78, 2008.

[9] M. Arezki and A. Benallal, "Fast adaptive filtering algorithm for acoustic noise cancellation," in Proceedings of the World Congress on Engineering 2012, vol. 2, London, UK, 2012.

[10] M. Mekarzia, "LATSI speech team of the institute of electronics of the university of blida," 2015-2016. 


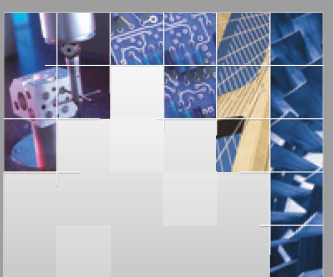

\section{Enfincering}
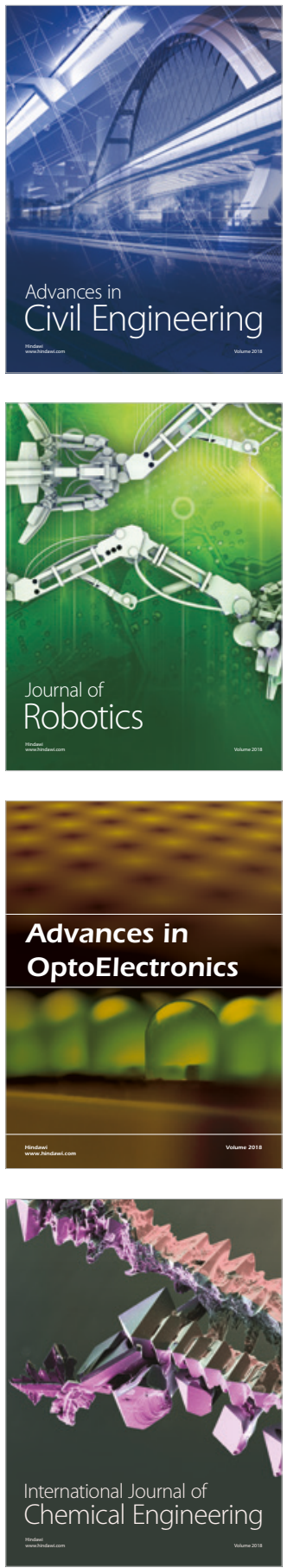

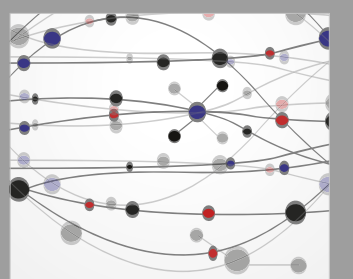

\section{Rotating \\ Machinery}

The Scientific World Journal

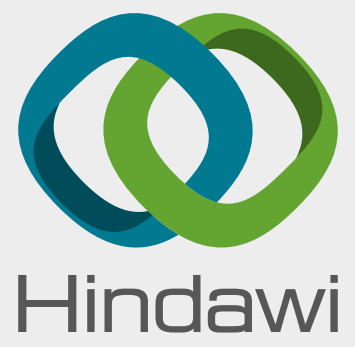

Submit your manuscripts at

www.hindawi.com
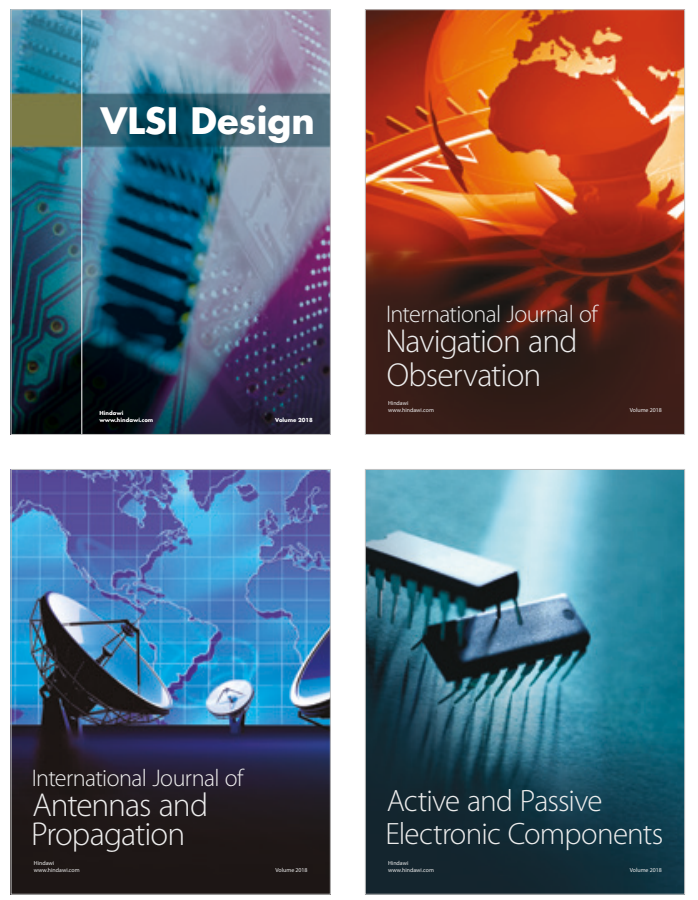
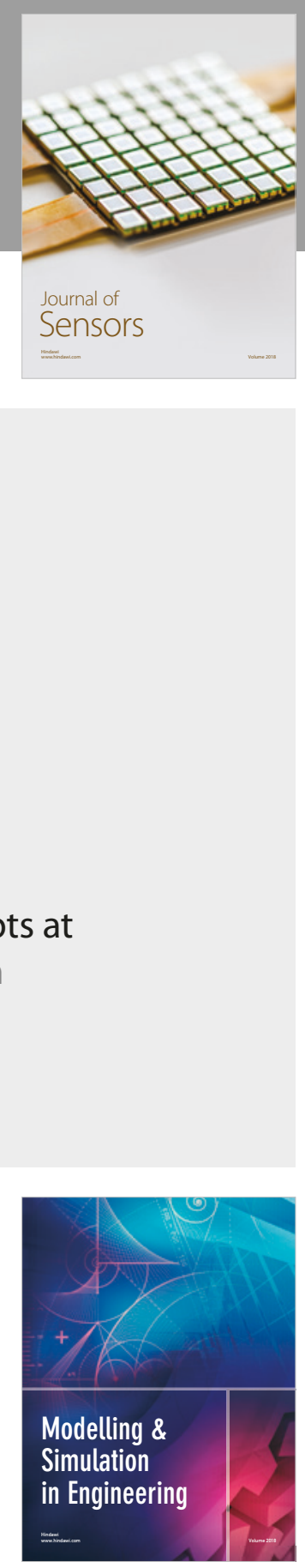

\section{Advances \\ Multimedia}
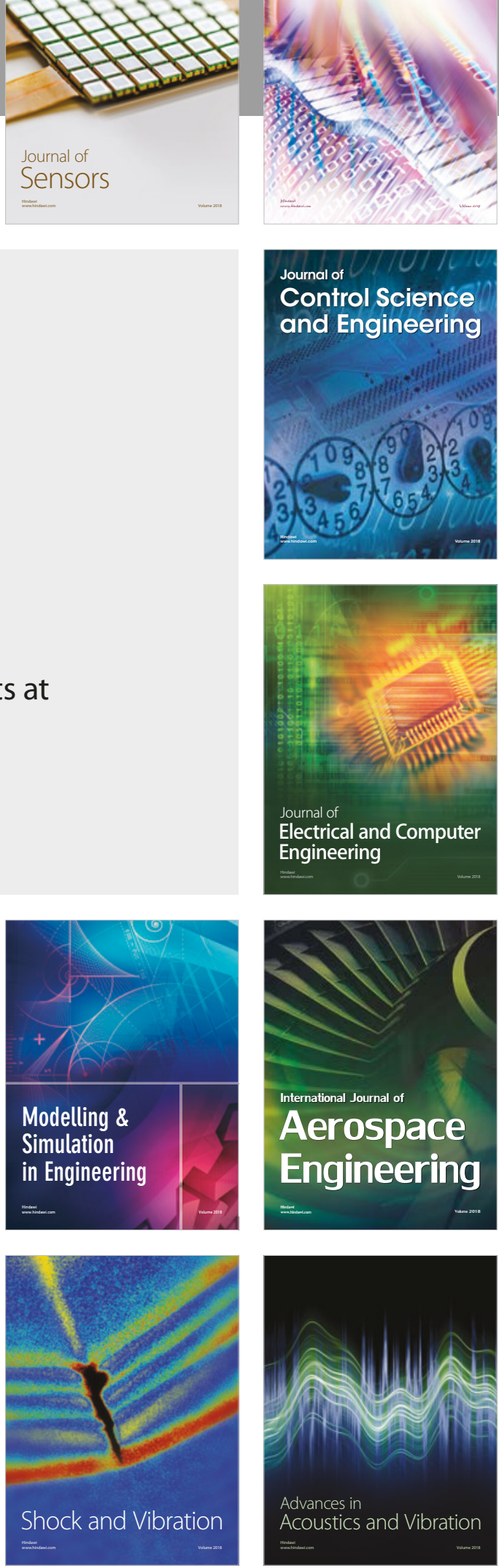